PROCEEDINGS OF THE

AMERICAN MATHEMATICAL SOCIETY

Volume 140, Number 1, January 2012, Pages 83-91

S 0002-9939(2011)10898-0

Article electronically published on May 19, 2011

\title{
RECOLLEMENTS FROM GENERALIZED TILTING
}

\author{
DONG YANG
}

(Communicated by Birge Huisgen-Zimmermann)

\begin{abstract}
Let $\mathcal{A}$ be a small dg category over a field $k$ and let $\mathcal{U}$ be a small full subcategory of the derived category $\mathcal{D} \mathcal{A}$ which generates all free $\operatorname{dg} \mathcal{A}$-modules. Let $(\mathcal{B}, X)$ be a standard lift of $\mathcal{U}$. We show that there is a recollement such that its middle term is $\mathcal{D} \mathcal{B}$, its right term is $\mathcal{D} \mathcal{A}$, and the three functors on its right side are constructed from $X$. This applies to the pair $(A, T)$, where $A$ is a $k$-algebra and $T$ is a good $n$-tilting module, and we obtain a result of Bazzoni-Mantese-Tonolo. This also applies to the pair $(\mathcal{A}, \mathcal{U})$, where $\mathcal{A}$ is an augmented dg category and $\mathcal{U}$ is the category of 'simple' modules; e.g., $\mathcal{A}$ is a finite-dimensional algebra or the Kontsevich-Soibelman $A_{\infty}$-category associated to a quiver with potential.
\end{abstract}

A recollement of triangulated categories is a diagram of triangulated categories and triangle functors

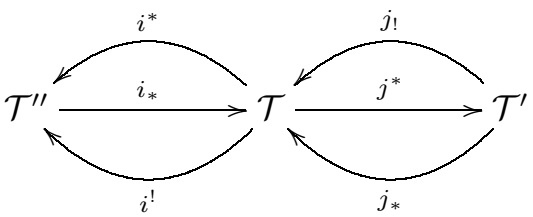

such that

- $\left(i^{*}, i_{*}, i^{!}\right)$and $\left(j_{!}, j^{*}, j_{*}\right)$ are adjoint triples;

- $i_{*}, j_{*}, j_{\text {! }}$ are fully faithful;

- $j^{*} \circ i_{*}=0$

- for every object $X$ of $\mathcal{T}$ there are two triangles

$i_{*} i^{!} X \longrightarrow X \longrightarrow j_{*} j^{*} X \longrightarrow$ and $j ! j^{*} X \longrightarrow X \longrightarrow i_{*} i^{*} X \longrightarrow$,

where the four morphisms are the units and counits.

We also say that this is a recollement of $\mathcal{T}$ in terms of $\mathcal{T}^{\prime}$ and $\mathcal{T}^{\prime \prime}$. This notion was introduced by Beilinson-Bernstein-Deligne in [4] in geometric contexts, where stratifications of varieties induce recollements of derived categories of sheaves.

In algebraic contexts, recollements are closely related to tilting theory. Let $A$ be a ring. Let $\mathcal{D}(A)=\mathcal{D}(\operatorname{Mod} A)$ denote the derived category of (right) $A$-modules, and $\operatorname{per} A$ denote the triangulated subcategory of $\mathcal{D}(A)$ generated by the free module of rank 1 . An object $T$ of per $A$ is called a partial tilting complex if $\operatorname{Hom}_{\mathcal{D}(A)}\left(T, \Sigma^{n} T\right)=$ 0 for $n \neq 0$, and a tilting complex if in addition $\operatorname{tria}(T)=\operatorname{per} A$, where $\operatorname{tria}(T)$ is the

Received by the editors June 21, 2010 and, in revised form, October 11, 2010 and November 8, 2010 .

2010 Mathematics Subject Classification. Primary 18E30, 16E45.

(C)2011 American Mathematical Society Reverts to public domain 28 years from publication 
triangulated subcategory of $\mathcal{D}(A)$ generated by $T$. Rickard's Morita theorem for derived categories states that the modified standard functors associated to a tilting complex $T$ over $A$ are triangle equivalences between $\mathcal{D}(A)$ and $\mathcal{D}\left(\operatorname{End}_{\mathcal{D}(A)}(T)\right)$; see [18]. Later in [12, Koenig proved that under certain conditions a partial tilting complex $T$ over $A$ yields a recollement of $\mathcal{D}(A)$ in terms of $\mathcal{D}\left(\operatorname{End}_{\mathcal{D}(A)}(T)\right)$ and a third derived category which measures how far the associated standard functors are from being equivalences (see also 8, 15]). In this sense, a recollement of derived categories can be viewed as a natural generalization of a derived equivalence. The relation between tilting theory and recollements of derived categories has been further studied in [1, 6]. The dg version of Rickard's theorem was developed by Keller in [10], and the result of Koenig was generalized to the dg setting by Jørgensen [9] and Nicolás-Saorín [17, where the role of partial tilting complexes is played by compact objects.

In this paper we deal with a situation which is 'dual' to the one in [12, [9, [17. Starting from a $\operatorname{dg}$ category $\mathcal{A}$ and a set of objets in the derived category $\mathcal{D} \mathcal{A}$ which generates all the compact objects, we construct a $\mathrm{dg}$ category $\mathcal{B}$ together with a recollement of $\mathcal{D B}$ in terms of $\mathcal{D} \mathcal{A}$ and another derived category; see Theorem 1 . We identify this third derived category with a certain known category in the special case when $\mathcal{A}$ is the Kontsevich-Soibelman $A_{\infty}$-category associated to a quiver with potential (Corollary 3 ) or when $\mathcal{A}$ is a finite-dimensional self-injective algebra (Corollary 4). The motivation for our study was to have a better understanding of the 'exterior' case of the Koszul duality (Corollary 2) and a result of BazzoniMantese-Tonolo which says that the right derived Hom-functor associated to an (infinitely generated) good tilting module is fully faithful (Corollary 1).

\section{The MAin RESUlt}

Let $k$ be a field and let $\mathcal{A}$ be a small dg $k$-category. Denote by $\operatorname{Dif} \mathcal{A}$ the $\operatorname{dg}$ category of (right) $\operatorname{dg} \mathcal{A}$-modules. A $\operatorname{dg} \mathcal{A}$-module $M$ is $\mathcal{K}$-projective if the dg functor $\operatorname{Dif} \mathcal{A}\left(M\right.$, ?) preserves acyclicity. For example, the free modules $A^{\wedge}=\operatorname{Dif} \mathcal{A}(?, A)$, $A \in \mathcal{A}$, are $\mathcal{K}$-projective. Let $\mathcal{D} \mathcal{A}$ denote the derived category of $\mathcal{A}$, which is triangulated with the suspension functor $\Sigma$ being the shift functor. For a set of objects or a subcategory $\mathcal{S}$ of $\mathcal{D} \mathcal{A}$ we denote by tria $\mathcal{S}$ the smallest triangulated subcategory of $\mathcal{D} \mathcal{A}$ containing all objects in $\mathcal{S}$ and closed under taking direct summands. Let $\operatorname{per} \mathcal{A}=\operatorname{tria}\left(A^{\wedge}, A \in \mathcal{A}\right)$. An object $M$ of $\mathcal{D} \mathcal{A}$ is compact if the functor $\mathcal{D} \mathcal{A}(M$, ?) commutes with infinite (set-indexed) direct sums or, equivalently, if $M$ belongs to $\operatorname{per} \mathcal{A}$. See 10 .

Let $\mathcal{U}$ be a full small subcategory of $\mathcal{D} \mathcal{A}$ such that

$$
\operatorname{tria} \mathcal{U} \supseteq \operatorname{per} \mathcal{A} \text {. }
$$

Let $(\mathcal{B}, X)$ be a standard lift of $\mathcal{U}([10$, Section 7$])$. Precisely, $\mathcal{B}$ is a dg subcategory of Dif $\mathcal{A}$ consisting of $\mathcal{K}$-projective resolutions over $\mathcal{A}$ of objects of $\mathcal{U}$ (to avoid confusion, for each object $B$ of $\mathcal{B}$ we will denote by $U_{B}$ the corresponding $\operatorname{dg} \mathcal{A}$ module) and $X$ is the $\operatorname{dg} \mathcal{B}^{o p} \otimes \mathcal{A}$-module defined by $X(B, A)=U_{B}(A)$. It induces a pair of adjoint dg functors and a pair of adjoint triangle functors

$$
\text { Dif } \mathcal{B} \underset{H_{X}}{\stackrel{T_{X}}{\rightleftarrows}} \text { Dif } \mathcal{A}, \quad \mathcal{D} \mathcal{B} \underset{\mathbf{R} H_{X}}{\stackrel{\mathbf{L} T_{X}}{\rightleftarrows}} \mathcal{D} \mathcal{A} .
$$


When $\mathcal{A}$ and $\mathcal{B}$ are dg $k$-algebras (i.e. $\operatorname{dg} k$-categories with one object), the functors $\mathbf{L} T_{X}$ and $\mathbf{R} H_{X}$ are usually written as $? \stackrel{\mathbf{L}}{\otimes} X$ and $\operatorname{RHom}(X, ?)$.

Let $X^{T}$ be the $\operatorname{dg} \mathcal{A}^{o p} \otimes \mathcal{B}$-module defined by

$$
X^{T}(A, B)=\operatorname{Dif} \mathcal{A}\left(X^{B}, A^{\wedge}\right),
$$

where for $B \in \mathcal{B}, X^{B}$ is by definition the $\operatorname{dg} \mathcal{A}$-module $X(B$, ?). From the definition of $X$ we see that $X^{B}=U_{B}$. The main result of this paper is

Theorem 1. Assume the notation as above. There is a $d g k$-category $\mathcal{C}$ and $a$ recollement of triangulated categories

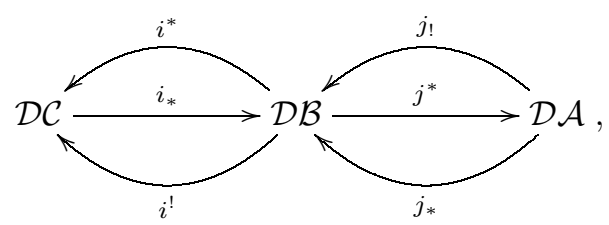

where the adjoint triple $\left(i^{*}, i_{*}, i^{!}\right)$is defined by a dg functor $F: \mathcal{B} \rightarrow \mathcal{C}$ (which is bijective on objects) such that $i_{*}=F^{*}: \mathcal{D C} \rightarrow \mathcal{D B}$ is the pull-back functor, and the adjoint triple $\left(j_{!}, j^{*}, j_{*}\right)$ is given by

$$
\begin{aligned}
& j_{!}=\mathbf{L} T_{X^{T}}, \\
& j^{*}=\mathbf{R} H_{X^{T}} \simeq \mathbf{L} T_{X}, \\
& j_{*}=\mathbf{R} H_{X} .
\end{aligned}
$$

Proof. In view of [17, Theorem 5], it suffices to prove

(a) $\mathbf{L} T_{X^{T}}$ is fully faithful,

(b) $\mathbf{R} H_{X^{T}} \simeq \mathbf{L} T_{X}$.

The proof for (a) is the same as the proof of [10, Lemma 10.5, the 'exterior' case c)]. Since $(\mathcal{B}, X)$ is a lift, the restriction of $\mathbf{L} T_{X}$ on the perfect derived category $\operatorname{per} \mathcal{B}$ is fully faithful, and its essential image is tria $\mathcal{U}$ (see [10, Section 7.3]):

$$
\left.\mathbf{L} T_{X}\right|_{\text {per } \mathcal{B}}: \operatorname{per} \mathcal{B} \stackrel{\sim}{\longrightarrow} \operatorname{tria} \mathcal{U} .
$$

It is clear that $\mathbf{R} H_{X}$ takes an object of tria $\mathcal{U}$ into per $\mathcal{B}$. Therefore, the restriction $\left.\mathbf{R} H_{X}\right|_{\text {tria } \mathcal{U}}$ is a quasi-inverse of $\left.\mathbf{L} T_{X}\right|_{\text {per } \mathcal{B}}$, and hence is fully faithful. It follows from [10, Lemma $6.2 \mathrm{a}$ )] that the restriction $\left.\mathbf{L} T_{X^{T}}\right|_{\text {per } \mathcal{A}}$ is naturally isomorphic to the restriction of $\left.\mathbf{R} H_{X}\right|_{\text {per } \mathcal{A}}$, which is fully faithful by condition (1). Condition (11) also implies that $\mathbf{R} H_{X}\left(A^{\wedge}\right)=\left(X^{T}\right)^{A}(A \in \mathcal{A})$ belongs to per $\mathcal{B}$ and hence is compact by [10, Theorem 5.3]. Now applying [10, Lemma $4.2 \mathrm{~b})$ ], we obtain that $\mathbf{L} T_{X^{T}}$ is fully faithful, finishing the proof of (a).

Let $Y \rightarrow X^{T}$ be a $\mathcal{K}$-projective resolution of $\operatorname{dg} \mathcal{A}^{o p} \otimes \mathcal{B}$-modules. Then the specialization $Y^{A} \rightarrow\left(X^{T}\right)^{A}$ is a $\mathcal{K}$-projective resolution of $\operatorname{dg} \mathcal{B}$-modules for any object $A$ of $\mathcal{A}$. Recall that $\left(X^{T}\right)^{A}$ is compact. It follows from [10, Lemma 6.2 a)] that $\mathbf{L} T_{Y^{T}} \simeq \mathbf{R} H_{Y}$. By [10, Lemma $6.1 \mathrm{~b}$ )], in order to prove $\mathbf{R} H_{X^{T}} \simeq \mathbf{L} T_{X}$, it suffices to prove that as $\operatorname{dg} \mathcal{B}^{o p} \otimes \mathcal{A}$-modules $Y^{T}$ and $X$ are quasi-isomorphic. Let $A \in \mathcal{A}$ and $B \in \mathcal{B}$. We have $H_{X}\left(U_{B}\right)=B^{\wedge}$, and hence

$$
\begin{aligned}
Y^{T}(A, B) & =\operatorname{Dif} \mathcal{B}\left(Y^{A}, B^{\wedge}\right) \\
& =\operatorname{Dif} \mathcal{B}\left(Y^{A}, H_{X}\left(U_{B}\right)\right) \\
& \cong \operatorname{Dif} \mathcal{A}\left(T_{X}\left(Y^{A}\right), U_{B}\right)
\end{aligned}
$$


The composition $T_{X}\left(Y^{A}\right) \rightarrow T_{X}\left(\left(X^{T}\right)^{A}\right)=T_{X} \circ H_{X}\left(A^{\wedge}\right) \rightarrow A^{\wedge}$ is exactly the counit $\mathbf{L} T_{X} \circ \mathbf{R} H_{X}\left(A^{\wedge}\right) \rightarrow A^{\wedge}$, which is an isomorphism in $\mathcal{D} \mathcal{A}$ because the restriction of $\mathbf{R} H_{X}$ on per $\mathcal{A}$ is fully faithful. Moreover, both $T_{X}\left(Y^{A}\right)$ and $A^{\wedge}$ are $\mathcal{K}$-projective $\operatorname{dg} \mathcal{A}$-modules. Therefore we have

$$
\begin{aligned}
Y^{T}(A, B) & \stackrel{q \cdot i s}{\leftarrow} \operatorname{Dif} \mathcal{A}\left(A^{\wedge}, U_{B}\right) \\
& =U_{B}(A) \\
& =X(A, B) .
\end{aligned}
$$

Further, every morphism in the above is functorial in both $A$ and $B$. This completes the proof of (b).

Corollary 1 ([3]). Let $A$ be a k-algebra and $n$ be a positive integer. Let $T$ be a good $n$-tilting module; i.e., $T$ is an $A$-module such that

(T1) the projective dimension of $T$ is less than or equal to $n$;

(T2) $\operatorname{Ext}_{A}^{i}\left(T, T^{(\alpha)}\right)=0$ for any integer $i>0$ and for any cardinal $\alpha$;

(T3) there is an exact sequence

$$
0 \longrightarrow A \longrightarrow T^{0} \longrightarrow T^{1} \longrightarrow \ldots \longrightarrow T^{n} \longrightarrow 0,
$$

where $T^{0}, \ldots, T^{n}$ are direct summands of direct sums of finite copies of $T$.

Put $B=\operatorname{End}_{A}(T)$. Then the right derived functor $\mathrm{RHom}_{A}(T, ?): \mathcal{D}(A) \rightarrow \mathcal{D}(B)$ is fully faithful, and $\mathcal{D}(A)$ is triangle equivalent to the triangle quotient of $\mathcal{D}(B)$ by the kernel of the left derived functor $? \otimes_{B} T$.

Proof. Let $\mathcal{U}$ be the full subcategory of $\mathcal{D}(A)$ consisting of one object $T$. Then the condition (T3) implies the condition (11). Let $X$ be a projective resolution of $T$ over $B^{o p} \otimes_{k} A$, and let $\tilde{B}$ be the $\operatorname{dg} k$-algebra $\operatorname{Dif} A(X, X)$. Then $X$ is $\mathcal{K}$-projective over $A$, and $(\tilde{B}, X)$ is a standard lift of $T$. Thanks to (T2), the representation map $B \rightarrow \tilde{B}$ of the $\operatorname{dg} B$-A-bimodule $X$ is a quasi-isomorphism, inducing mutually quasi-inverse triangle equivalences ? $\stackrel{\mathbf{L}}{\otimes_{\tilde{B}}} \tilde{B}=\operatorname{RHom}_{\tilde{B}}(\tilde{B}, ?): \mathcal{D}(\tilde{B}) \rightarrow \mathcal{D}(B)$ and $? \stackrel{\mathrm{L}}{\otimes_{B}} \tilde{B}: \mathcal{D}(B) \rightarrow \mathcal{D}(\tilde{B})$. Now applying Theorem 1 and composing the resulting recollement with the above triangle equivalences, we obtain a recollement

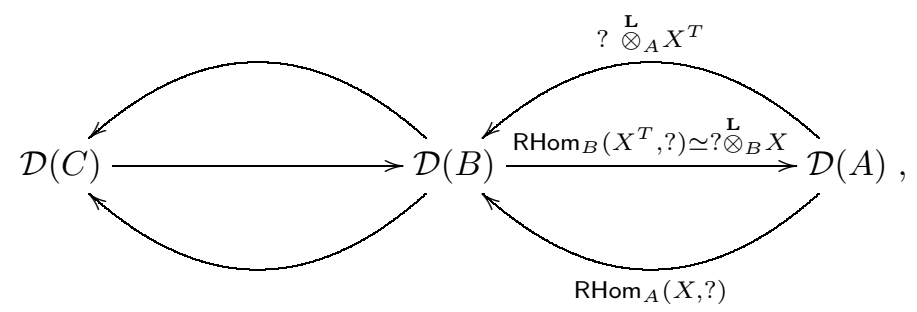

where $C$ is a $\operatorname{dg} k$-algebra. Since $X$ and $T$ are quasi-isomorphic as $\operatorname{dg} B^{o p} \otimes_{k} A$ modules, we have natural isomorphisms $? \stackrel{\mathrm{L}}{\otimes_{B}} X \simeq ? \stackrel{\mathrm{L}}{\otimes_{B}} T$ and $\operatorname{RHom}_{A}(X, ?) \simeq$ $\mathrm{RHom}_{A}(T, ?)([10$, Lemma $\left.6.1 \mathrm{~b})]\right)$. The desired result follows at once.

Remark. a) This result is due to Bazzoni [2] for $n=1$ and Bazzoni-ManteseTonolo [3] for general $n$ for all rings $A$. 
b) By Theorem [1 the left half of the recollement in the proof is induced from a dg homomorphism $B \rightarrow C$. For the case $n=1$ and for all rings $A$, Chen and $\mathrm{Xi}$ obtained in [6] such a recollement with $C$ being an ordinary ring (so that the map $B \rightarrow C$ becomes a homomorphism of rings). They used some results in [1] and many other results such as the homological properties of the tilting module $T$.

To state the next corollary, we need to introduce some notions. Let $\mathcal{A}$ be an augmented dg $k$-category ([10, Section 10.2]); i.e.,

- distinct objects of $\mathcal{A}$ are nonisomorphic,

- for each $A \in \mathcal{A}$, a dg module $\bar{A}$ is given such that $H^{0} \bar{A}(A) \cong k$ and $H^{n} \bar{A}\left(A^{\prime}\right)$ whenever $n \neq 0$ or $A^{\prime} \neq A$.

Let $\left(\mathcal{A}^{*}, X\right)$ be a standard lift of $\mathcal{U}=\{\bar{A} \mid A \in \mathcal{A}\} \subset \mathcal{D} \mathcal{A}$. By abuse of language, we call the $\operatorname{dg} k$-category $\mathcal{A}^{*}$ the $\operatorname{Koszul} d u a l$ of $\mathcal{A}$. Assume that the condition (11) holds; e.g., this happens in the 'exterior' case in [10, Section 10.5].

Corollary 2. Assume the notation as above. There is a recollement of derived categories of $d g k$-categories

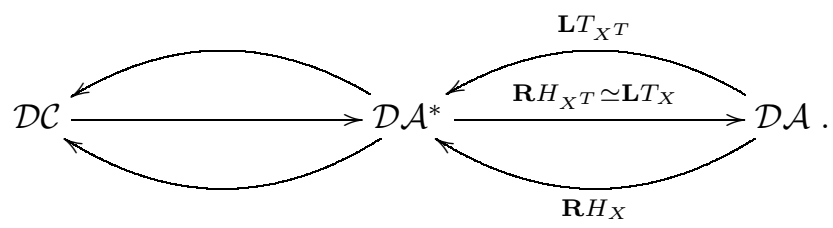

Proof. This is a direct consequence of Theorem 1.

\section{THE LEFT TERM}

As in the preceding section, we let $k$ be a field, $\mathcal{A}$ be a small $\operatorname{dg} k$-category, $\mathcal{U}$ be a full small subcategory of the derived category $\mathcal{D} \mathcal{A}$ such that tria $\mathcal{U} \supseteq$ per $\mathcal{A}$, and let $(\mathcal{B}, X)$ be a standard lift of $\mathcal{U}$. Theorem 11 says that there is a recollement of $\mathcal{D B}$ in terms of $\mathcal{D} \mathcal{A}$ and a third derived category $\mathcal{D C}$, where $\mathcal{C}$ is a $\mathrm{dg} k$-category whose objects are in bijection with the objects of $\mathcal{U}$.

Let $\mathcal{V}=\left\{\left(X^{T}\right)^{A} \mid A \in \mathcal{A}\right\} \subset \mathcal{D B}$. From the proof of the theorem we obtain a commutative diagram

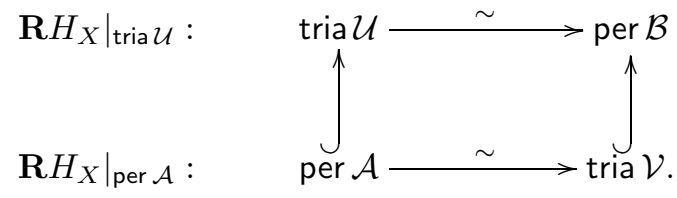

Therefore $\mathrm{RH}_{X}$ induces a triangle equivalence between the triangle quotient categories

$$
\operatorname{tria} \mathcal{U} / \operatorname{per} \mathcal{A} \sim \operatorname{per} \mathcal{B} / \operatorname{tria} \mathcal{V}
$$

For a triangulated category $\mathcal{T}$, let $\mathcal{T}^{c}$ denote the subcategory of compact objects in $\mathcal{T}$. Let Tria $\mathcal{V}$ be the localizing subcategory of $\mathcal{D B}$ generated by the objects in $\mathcal{V}$. We have $(\mathcal{D B})^{c}=\operatorname{per} \mathcal{B}$ and $(\operatorname{Tria} \mathcal{V})^{c}=\operatorname{tria} \mathcal{V}$. Thus by [16. Theorem 2.1], the category $\left(\mathcal{D B} / \text { Tria }_{\mathcal{V}}\right)^{c}$ is triangle equivalent to the idempotent completion of per $\mathcal{B} /$ tria $\mathcal{V}$. 
Since the essential image of $\mathbf{L} T_{X^{T}}$ is exactly Tria $\mathcal{V}$, it follows that $\mathcal{D C}$ is triangle equivalent to the triangle quotient $\mathcal{D B} /$ Tria $\mathcal{V}$ and hence is an 'unbounded version' of tria $\mathcal{U} / \operatorname{per} \mathcal{A} \cong \operatorname{per} \mathcal{B} / \operatorname{tria} \mathcal{V}$. Apparently, $\mathcal{D C}$ vanishes if and only if tria $\mathcal{U} / \operatorname{per} \mathcal{A}$ does as well, in which case $\mathcal{U}$ consists of a set of compact generators for $\mathcal{D} \mathcal{A}$.

In the following two special cases, we are able to identify $\mathcal{D C}$ with a certain known category (however, the $\mathrm{dg}$ category $\mathcal{C}$ is not easy to describe).

Corollary 3. Let $(Q, W)$ be a quiver with potential. Let $\mathcal{A}_{(Q, W)}$ be the KontsevichSoibelman $A_{\infty}$-category ([13, Section 3.3]) (or its enveloping dg category), let $\widehat{\Gamma}_{(Q, W)}$ be the complete Ginzburg dg category ([7, Section 5]), and let $\widetilde{C}_{(Q, W)}$ be the 'unbounded version' of the generalized cluster category ([11, Remark 4.1]). Then there is a recollement of triangulated categories

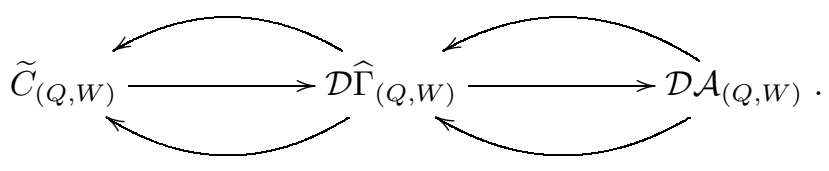

Proof. Let $\mathcal{A}=\mathcal{A}_{(Q, W)}$ and let $\mathcal{U}$ be the category of simple $\mathcal{A}$-modules. Then condition (11) holds since $\mathcal{A}$ is finite-dimensional, and there is a standard lift ( $\mathcal{B}, X)$ such that the dg category $\Gamma=\widehat{\Gamma}_{(Q, W)}$ (as the Koszul dual of $\left.\mathcal{A}_{(Q, W)}\right)$ is quasiisomorphic to $\mathcal{B}$. By Corollary 2 , there is a recollement with the middle term being $\mathcal{D} \Gamma$, the right term being $\mathcal{D} \mathcal{A}$, and the right upper functor being $\mathbf{L} T_{X^{T}}$. It remains to prove that the left term of this recollement is triangle equivalent to $\widetilde{\mathcal{C}}_{(Q, W)}$. Object sets of $\mathcal{A}$, of $\mathcal{U}$, and of $\Gamma$ can all be identified with the vertex set $Q_{0}$ of the quiver $Q$. For a vertex $i$ of $Q$, considered as an object of $\mathcal{A}$, the right $\mathrm{dg}$ $\Gamma$-module $\left(X^{T}\right)^{i}$ is isomorphic in $\mathcal{D} \Gamma$ to $\Sigma^{-3} S_{i}$, where $S_{i}$ is the simple top of the free $\Gamma$-module $i^{\wedge}$. Thus the essential image of $\mathbf{L} T_{X^{T}}$ is the localizing subcategory $\mathcal{D}_{0} \Gamma=\operatorname{Tria}\left(S_{i}, i \in Q_{0}\right)$ of $\mathcal{D} \Gamma$ generated by the $S_{i}, i \in Q_{0}$. Thus the left term of the recollement is triangle equivalent to the triangle quotient $\mathcal{D} \Gamma / \mathcal{D}_{0} \Gamma$, which is by definition $\widetilde{\mathcal{C}}_{(Q, W)}$.

Let $A$ be a finite-dimensional basic $k$-algebra. Let $S$ be the direct sum of the objects in a set of representatives of isomorphism classes of simple $A$-modules, and let $X$ be a projective resolution of $S$. Then $A^{*}=\operatorname{Dif} A(X, X)$ is the Koszul dual of $A$.

Corollary 4 ([14]). Let $A$ be a finite-dimensional basic self-injective $k$-algebra,

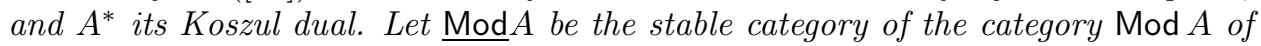
$A$-modules. Then there is a recollement of triangulated categories

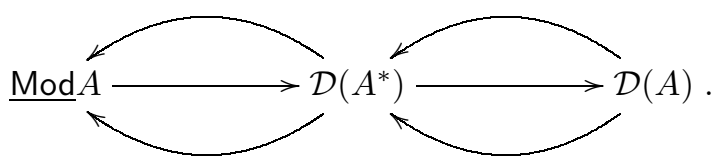

Proof. Let $\bmod A$ be the category of finite-dimensional $A$-modules and $\bmod A$ its stable category. As a triangulated subcategory of $\mathcal{D}(A)$, the bounded derived category $\mathcal{D}^{b}(\bmod A)$ of $\bmod A$ coincides with tria $S$. Recall that the essential image of 
$? \stackrel{\mathbf{L}}{\otimes_{A}} X^{T}$ is Tria $X^{T}$. Consider the following commutative diagram:

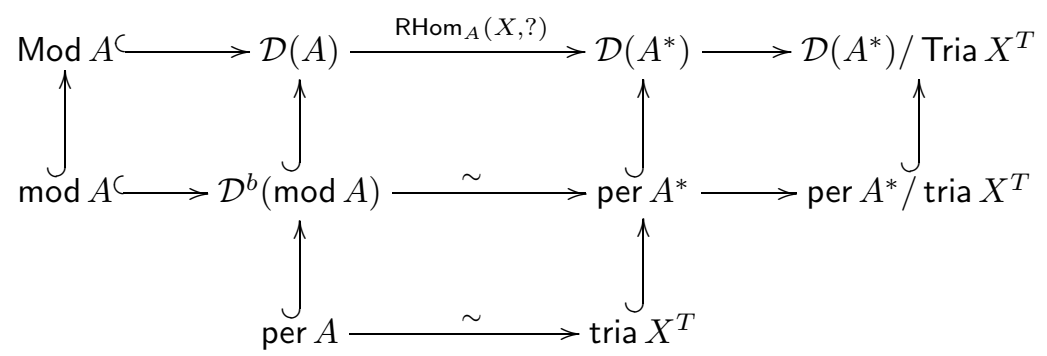

where the leftmost horizontal functors are the canonical embeddings, and the rightmost horizontal functors are the canonical projections. The restriction of $\mathrm{RHom}_{A}(X, ?)$ on Mod $A$ commutes with infinite direct sums, because $X$ can be chosen such that its component in each degree is a finitely generated projective $A$ module. Therefore the composition of the three functors in the first row, denoted by $F$, commutes with infinite direct sums. Since $\mathrm{RHom}_{A}(X, A) \cong X^{T}$ belongs to Tria $X^{T}$, it follows that $F$ factors through the stable category $\underline{\operatorname{Mod}} A$. In this way, we obtain a triangle functor

$$
\bar{F}: \underline{\operatorname{Mod}} A \rightarrow \mathcal{D}\left(A^{*}\right) / \operatorname{Tria} X^{T},
$$

which commutes with infinite direct sums. It is known that $\operatorname{Mod} A$ is compactly generated by $\underline{\bmod } A$ and $(\underline{\operatorname{Mod}} A)^{c}=\underline{\bmod } A$. Moreover, the restriction $\left.\bar{F}\right|_{\bmod A}$ is the composition of the following three functors:

$$
\underline{\bmod } A \longrightarrow \mathcal{D}^{b}(\bmod A) / \operatorname{per} A \stackrel{\sim}{\longrightarrow} \operatorname{per} A^{*} / \operatorname{tria} X^{T} \longrightarrow \mathcal{D}\left(A^{*}\right) / \operatorname{Tria} X^{T} .
$$

The first functor is also an equivalence ([19, Theorem 2.1]). Therefore $\bar{F}$ induces a triangle equivalence between $\underline{\bmod A}=(\underline{\operatorname{Mod}} A)^{c}$ and $\operatorname{per} A^{*} / \operatorname{tria} X^{T}=$ $\left(\mathcal{D}\left(A^{*}\right) / \operatorname{Tria} X^{T}\right)^{c}$. By [10, Lemma 4.2], $\bar{F}$ itself is an equivalence. Now applying Corollary 2 we obtain the desired recollement.

Remark. Let $\mathcal{H}(\operatorname{Inj} A)$ be the homotopy category of injective $A$-modules and $\mathcal{H}_{a c}(\operatorname{Inj} A)$ be its full subcategory of acyclic complexes. Applying a result of Krause [14, Corollary 4.3] to the algebra $A$, we obtain a recollement of $\mathcal{H}(\operatorname{Inj} A)$ in terms of $\mathcal{D}(A)$ and $\mathcal{H}_{a c}(\operatorname{Inj} A)$ with the right middle functor being the canonical projection $Q: \mathcal{H}(\operatorname{Inj} A) \rightarrow \mathcal{D}(A)$. We claim that this recollement is equivalent to the one in Corollary 4. Indeed, Krause proved in [14] that $\mathcal{H}(\operatorname{Inj} A)$ is compactly generated by (an injective resolution of) the $A$-module $S$ and that there is a triangle

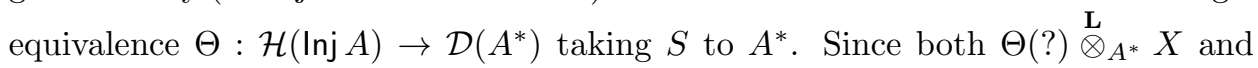
$Q$ commute with infinite direct sums and $\Theta(S) \stackrel{\mathbf{L}}{\otimes}_{A^{*}} X \cong X \cong S$, it follows that they are isomorphic. Namely, the right middle parts of the two recollements are equivalent via the equivalence $\Theta$. Therefore the two recollements are equivalent.

Now let us construct the equivalence $\Theta$ by sketching the proof of the assertion that $\mathcal{H}(\operatorname{Inj} A)$ and $\mathcal{D}\left(A^{*}\right)$ are triangle equivalent. Let $\operatorname{Dif}_{\operatorname{lnj}} A$ be the full $\operatorname{dg}$ subcategory of Dif $A$ consisting of complexes of injective $A$-modules, let $\mathbf{i} S$ be an injective resolution of the $A$-module $S$, and put $B=\operatorname{Dif} A(\mathbf{i} S, \mathbf{i} S)$. Then the $\operatorname{dg}$ $B^{o p} \otimes A^{*}$-module Dif $A(X, \mathbf{i} S)$ yields a triangle equivalence $\Phi: \mathcal{D}(B) \rightarrow \mathcal{D}\left(A^{*}\right)$ (see [10, Section 7.3]). Moreover, $\operatorname{Dif}_{\operatorname{Inj}} A$ is a $\operatorname{dg}$ enhancement of the triangulated 
category $\mathcal{H}(\operatorname{Inj} A)$ in the sense of Bondal-Kapranov [5], and there is a dg functor $\operatorname{Dif}_{\text {Inj }} A\left(\mathbf{i} S\right.$, ?) $: \operatorname{Dif}_{\operatorname{lnj}} A \rightarrow \operatorname{Dif} B$. Taking zeroth comhomologies gives us a triangle functor $\mathcal{H}(\operatorname{Inj} A) \rightarrow \mathcal{H}(B)$, and composing it with the canonical projection $\mathcal{H}(B) \rightarrow \mathcal{D}(B)$ we obtain a triangle equivalence $\Psi: \mathcal{H}(\operatorname{Inj} A) \rightarrow \mathcal{D}(B)$ (cf. the proof of [10, Theorem 4.3]). Now the composition $\Theta=\Phi \circ \Psi: \mathcal{H}(\operatorname{Inj} A) \rightarrow \mathcal{D}\left(A^{*}\right)$ is a triangle equivalence, which takes i $S$ to $A^{*}$ (up to isomorphism), as desired.

\section{ACKNOWLEDGEMENTS}

The author gratefully acknowledges financial support from the Max-PlanckInstitut für Mathematik in Bonn. He thanks Pedro Nicolás, Steffen Koenig and the referee for some helpful remarks on previous versions. Part of the paper was written during the author's visit to the Department of Mathematics at Shanghai Jiaotong University. He thanks Guanglian Zhang for his warm hospitality.

\section{REFERENCES}

[1] Lidia Angeleri Hügel, Steffen Koenig and Qunhua Liu, Recollements and tilting objects, J. Pure Appl. Algebra 215 (2011), no. 4, 420-438.

[2] Silvana Bazzoni, Equivalences induced by infinitely generated tilting modules, Proc. Amer. Math. Soc. 138 (2010), no. 2, 533-544. MR2557170 (2011a:16012)

[3] Silvana Bazzoni, Francesca Mantese and Alberto Tonolo, Derived equivalence induced by $n$-tilting modules, arXiv:0905.3696, to appear in Proc. Amer. Math. Soc.

[4] Alexander A. Beilinson, Joseph Bernstein and Pierre Deligne, Analyse et topologie sur les espaces singuliers, Astérisque, vol. 100, Soc. Math. France, 1982 (French). MR751966 (86g:32015)

[5] Aleksei I. Bondal and Mikhail M. Kapranov, Enhanced triangulated categories, Mat. Sb. 181 (1990), no. 5, 669-683; translation in Math. USSR-Sb. 70 (1991), no. 1, 93-107. MR.1055981 (91g:18010)

[6] Hongxing Chen and Changchang Xi, Good tilting modules and recollements of derived module categories, arXiv:1012.2176.

[7] Victor Ginzburg, Calabi-Yau algebras, arXiv:math/0612139v3 [math.AG].

[8] Dieter Happel, Partial tilting modules and recollement, Proceedings of the International Conference on Algebra, Part 2 (Novosibirsk, 1989), 345-361, Contemp. Math. 131, Part 2, Amer. Math. Soc., Providence, RI, 1992. MR.1175843 (93k:16011)

[9] Peter Jørgensen, Recollement for Differential Graded Algebras, J. Algebra 299 (2006), 589601. MR2228328 (2007b:16023)

[10] Bernhard Keller, Deriving DG categories, Ann. Sci. École Norm. Sup. (4) 27 (1994), no. 1, 63-102. MR.1258406 (95e:18010)

[11] Bernhard Keller and Dong Yang, Derived equivalences from mutations of quivers with potential, Adv. Math. 226 (2011), no. 3, 2118-2168.

[12] Steffen Koenig, Tilting complexes, perpendicular categories and recollements of derived categories of rings, J. Pure Appl. Algebra 73 (1991), 211-232. MR.1124785 (92k:18009)

[13] Maxim Kontsevich and Yan Soibelman, Stability structures, motivic Donaldson-Thomas invariants and cluster transformations, arXiv:0811.2435.

[14] Henning Krause, The stable derived category of a Noetherian scheme, Compos. Math. 141 (2005), no. 5, 1128-1162. MR2157133 (2006e:18019)

[15] Jun-Ichi Miyachi, Recollements and tilting complexes, J. Pure Appl. Algebra 183 (2003), 245-273. MR1992048 (2004g:16007)

[16] Amnon Neeman, The connection between the K-theory localisation theorem of Thomason, Trobaugh and Yao, and the smashing subcategories of Bousfield and Ravenel, Ann. Sci. École Normale Supérieure 25 (1992), 547-566. MR.1191736 (93k:18015)

[17] Pedro Nicolás and Manuel Saorín, Parametrizing recollement data for triangulated categories, J. Algebra 322 (2009), no. 4, 1220-1250. MR2537682 (2010g:18015) 
[18] Jeremy Rickard, Morita theory for derived categories, J. London Math. Soc. 39 (1989), 436456. MR:1002456 (91b:18012)

[19] Jeremy Rickard, Derived categories and stable equivalence, J. Pure and Appl. Algebra 61 (1989), 303-317. MR1027750 (91a:16004)

MaX-Planck-Institut für Mathematik in Bonn, Vivatsgasse 7, 53111 Bonn, Germany

Current address: HIM, Hausdorff Research Institute for Mathematics, Poppelsdorff Allee 82, D-53115, Bonn, Germany

E-mail address: yangdong98@mails.thu.edu.cn 